\title{
Advancement in infotainment system in automotive sector with vehicular cloud network and current state of art
}

\author{
Reshma S. ${ }^{1}$, Chetanaprakash ${ }^{2}$ \\ ${ }^{1}$ Department of Computer Science and Engineering, Global Academy of Technology, Bengaluru, India \\ ${ }^{2}$ Department of Computer Science and Engineering, Bapuji Institute of Engineering and Technology, Davangere, India
}

\begin{tabular}{|c|c|}
\hline Article Info & ABSTRACT \\
\hline Article history: & \multirow{10}{*}{$\begin{array}{l}\text { The automotive industry has been incorporating various technological } \\
\text { advancement on top-end versions of the vehicle order to improvise } \\
\text { the degree of comfortability as well as enhancing the safer driving system. } \\
\text { Infotainment system is one such pivotal system which not only makes } \\
\text { the vehicle smart but also offers abundance of information as well as } \\
\text { entertainment to the driver and passenger. The capability to offer extensive } \\
\text { relay of service through infotainment system is highly dependent on } \\
\text { vehicular adhoc network as well as back end support of cloud environment. } \\
\text { However, it is know that such legacy system of vehicular adhoc network is } \\
\text { also characterized by various problems associated with channel capacity, } \\
\text { latency, heterogeneous network processing, and many more. Therefore, this } \\
\text { paper offers a comprehensive insight to the research work being carried out } \\
\text { towards leveraging the infotainment system in order to obtain the true picture } \\
\text { of strength, limitation, and open end problems associated with infotainment } \\
\text { system. }\end{array}$} \\
\hline Received Apr 25, 2019 & \\
\hline Revised Oct 18, 2019 & \\
\hline Accepted Nov 3, 2019 & \\
\hline Keywords: & \\
\hline Cloud & \\
\hline Infotainment system & \\
\hline Internet of vehicle & \\
\hline Vehicular adhoc network & \\
\hline Vehicular network & \\
\hline
\end{tabular}

Copyright $(2020$ Institute of Advanced Engineering and Science. All rights reserved.

\section{Corresponding Author:}

Reshma S.,

Department of Computer Science and Engineering,

Global Academy of Technology,

Bengaluru, India.

Email: reshmas@gat.ac.in

\section{INTRODUCTION}

With the advancement of the technology in the automotive sector, various innovations have started evolving in recent times [1]. Such innovations are essentially meant for enhancing the safety of the vehicle driving on road, optimizing performance of engine, managing different forms of automated in-built system, and offering infotainment [2]. Out of all these, the current topic of discussion is basically the infotainment system which combines both software and hardware in order to provide various information as well as entertainment [3]. Apart from this, navigational assistance and health-diagnostics of vehicle are also included in the infotainment system assisting in comfortable and safer driving experience [4]. Infotainment system is a topic worth discussion as it is found that there will be a tremendous increase of its usage by the year 2022 . With the evolution of different commercial products, it can be said that there are certain premium luxury cars which in equipped with massive computational capability and is capable of generating massive size of data each seconds [5]. With the evolution of cloud computing and Internet-of-Things, there is a rise of multiple smart devices that are connected to each other with more mobility services supportability [6]. Similar trend of device-to-device connectivity paradigm is being initiated by various automakers. This gives a rise of internetof-vehicles [7] where the vehicles are treated as a mobile node and is connected using cloud system. Basically, internet of vehicle is an advanced version of vehicle to vehicle communication. Such forms of communication scheme make use of geo-positioning system, sensors, braking system, entertainment system, etc. Hence, such smart vehicles are equipped with artificial intelligence. Generally, adhoc network is used for 
establish communication among the vehicles with infotainment system and then they could be connected via World Wide Web. Usage of $5 \mathrm{G}$ communication system also facilitates such forms of communication system. However, it is not that simple in reality. In fact there are only countable numbers of vehicles that offer such services as the devices, infrastructures as well as services are pretty expensive in nature. Therefore, such applications are few to find in all the countries or accessible to common people. However, demands of the clients are changing with the evolution of new technologies which is more pervasive or ubiquitous in its form [8]. There are multiple reason that results in not so proliferation of using infotainment system over vehicular network viz. i) the first reason is the roadways, network, and transportation infrastructure which has to be changed accordingly. Road should be equipped with hotspots called as road-side unit and all road-side units should be connected to some core network connected with cloud or internet, ii) infotainment system should have integrated with maximum sensors and internal system of the vehicle in order to offer correct information for safer driving. Infotainment system is a part of on-board unit which should also offer extensive communication supportability of almost all forms of wireless standards e.g. Bluetooth, Near-Field Communication, Wi-Fi, GSM, CDMA, etc. Various studies e.g. [9-12] shows that vehicular adhoc network suffers significant problems and the same problem do exists in vehicular cloud networks too [13].

Therefore, this paper discusses about the research work carried out towards infotainment system when using cloud-based vehicular adhoc network. The paper also offers insights towards effectiveness and limitation of existing infotainment system. The organization of the paper is as follow Section 2 discusses about the basic fundamentals of the infotainment system followed by discussion of existing research contribution in Section 3. The briefing of the open research issues is carried out in Section 4 while conclusion of the paper is briefed in Section 5.

\section{INSIGHTS ON INFOTAINMENT SYSTEM}

Basically, an Infotainment system is a system that integrates the entertainment system as well as data services in order to facilitate the better form of driving experience. A typical infotainment system is shown in Figure 1 which consists of audio as well as video interfaces, keypads, and touchscreen. At present, the availability of infotainment system is restricted only to some premium cars but it is expected to have $80 \%$ increment in the usage of infotainment system by the year 2025 [14].

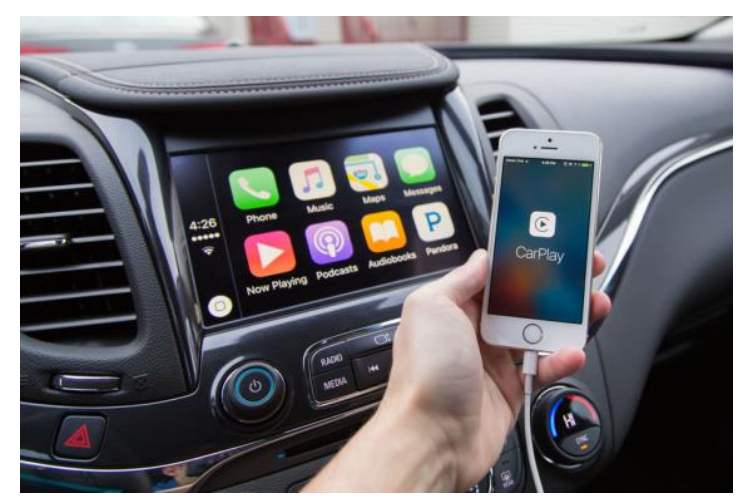

Figure 1. Apple car play infotainment system

Basically, infotainment system is a safer and smarter mechanism to utilize the smartphones and it allows the driver to perform all the operation on the visual interface of the infotainment system which they can do in their smartphone. Various third party applications (e.g. Apple's digital assistance, Siri, etc) are also utilized with the infotainment system for improving the features. At present, they can be controlled by knob, touch screen, and voice-based commands. Hence, infotainment system offers the capability to connect the complete vehicle with a network as well as a vehicle can also act as a hotspot for other vehicle. The degree of smartness of infotainment system can be further improved by integrating vehicular adhoc network and cloud environment. It is potentially important to ensure higher efficiency, minimal delay, reliability, scalability, as well as security when an infotainment system is connected with vehicular adhoc network system. Such forms of incorporation of operation incorporate in smartness in offering multiple services though infotainment giving a shape of intelligent transportation system. Therefore, appending cloud with the vehicular adhoc network permits amalgamation of various conventional computing systems with 
the available resources of the vehicle. However, there is a lot of difference between typical cloud environment and vehicular cloud as shown in Figure 2. In vehicular cloud, there are various contributor e.g. gateway system, infrastructure, and broker. Basically, vehicular cloud system is classified into three types viz. a) vehicular cloud, b) vehicle using cloud, and c) hybrid system [15]. Basically, typical cloud system emphasizes on clients, application, platform, and infrastructure in the form of services. However, vehicular system using cloud will use multiple components connected with specific cloud-based services. The vehicular nodes are integrated with the clients while different forms of applications used either in smartphone or the infotainment system is connected with application services on cloud. Different application could be associated with both information as well as entertainment which utilize various cloud-based applications. Similarly, usage of on-board units using radio frequencies for communication is connected with the platform-based services on cloud. Finally, utilization of on-board units and road-side units are integrated with the infrastructure based cloud services.

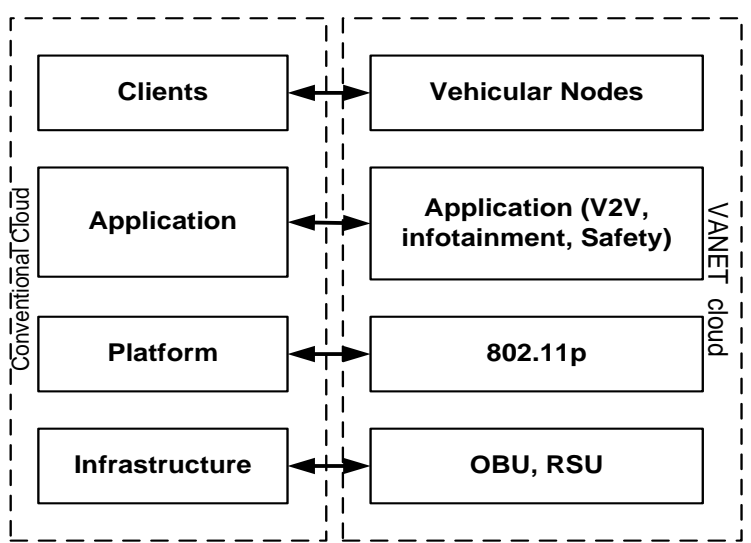

Figure 2. Difference between cloud and cloud with vehicular network

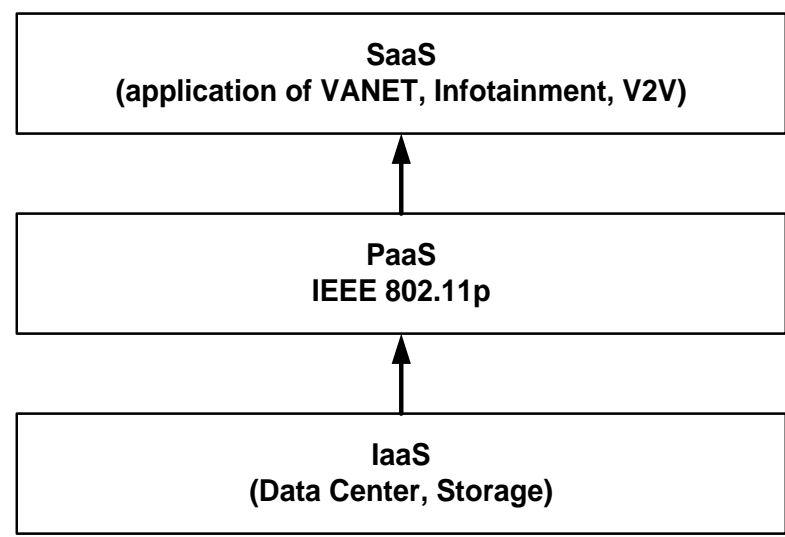

Figure 3. Vehicular cloud service architecture

The conventional vehicular cloud service architecture (Figure 3) consists of only three layers. The bottom layer is Infrastructure as a Service which is meant for storage, connectivity with data center etc. The middle layer is for Platform as a Service which is integrating with the conventional IEEE standard of vehicular adhoc network. The top layer is for Software as a Service which is basically meant for infotainment system as well as vehicle-to-vehicle communication, as well as different other application of vehicular cloud system.

\section{EXISTING RESEARCH CONTRIBUTION}

From the discussion made in prior section, it can be seen that there are various forms of network services being run within an infotainment system of a vehicle. Network plays a very significant role in the process of relaying services. At present, there are various research work being carried out towards leveraging infotainment system over a vehicular network and this section briefs of all the significant literature addressing the problem. The existing system has been found to be classified into two core methods towards development of infotainment system which is discussed as below:

\subsection{Infotainment system over vehicular networks}

The betterment of services offered in infotainment system can be only facilitated by as faster wireless communication system. Radio over Fiber (RoF) based approach is proven to for faster communication with the road-side units harnessing the concept of IoV which is also characterized by multiple interfaces of communication system. This approach was seen in the work of Sherazi et al. [16] where multiple vehicles perform group-based communication under defined radio access units. All the access units are integrated with a control system that uses optical fiber to establish connection with its backbone cloud network. Multiple test environments are used for assessing its outcome with respect to throughput, load, delay, etc. Usage of open source is also carried out towards developing an interface application of infotainment system. The main idea behind adding such interface is that existing design of infotainment system are developed on different platform and hence is always dependent on specific form of application to

\footnotetext{
Advancement in infotainment system in automotive sector with vehicular cloud network and ... (Reshma S.)
} 
be executed within it. Therefore, the work of Kovacevic et al. [17] proved this concept by using java toward building an application interface followed by developing a prototype application. Apart from the problem of data forwarding and service transition, it was seen distribution of data in large scale, mobility, and sensitivity to delay is another set of problems in infotainment system. According to Guo et al. [18], all these problems can be sorted by compressed sensing methods. This method is reported to assists in video streaming, recognition of object, sensing vehicle crowd, security and privacy protection, estimation of road traffic system, tracking object, etc. However, they are too associated with various challenges e.g. hardware design, analysis of behaviour on-road, streaming data, etc. Service relaying over infotainment system is also facilitated by using Wi-Fi protocol, however, it suffers from interference problem and can significantly affect the data to be received in infotainment device. Study towards this direction is carried out by Na et al. [19] has discussed various approaches to control interference problems e.g. channel hoping approach, power control policy, infra-structured based cooperation with adjacent nodes, etc. Authors have presented a simulationbased study with two different case studies to solve interference problems. The study outcome shows that presented scheme offers better throughput. There are some studies when conventional approaches have been used quite uniquely to offer better infotainment services. It has been reported that usage of television bands can facilitate better relaying of service. The work carried out by Chen et al. [20] has specified that reusing the spatial spectrum can assist in relaying data for infotainment services. However, the only difference it makes with other conventional approach is that-conventional approaches data is restricted to core internet network (i.e. cloud) whereas the presented scheme forwards the data to application server and geo-location database server. The work carried out by Rene et al. [21] have emphasized on adoption of emulation towards investigating infotainment system associated with the vehicular network. The authors have discussed a real-time emulation platform called as Vespa for this purpose and discussed various analytical capabilities of this emulator. Different from main-stream studies, there is a study conducted by Tian et al. [22] that discusses about the problem associated with the position of display for the infotainment device. The complete study has been carried out considering multiple display position to find that fluctuation on the position of lane could be minimize considering position with cluster display. The multiple display position considered was on radio interface, phone interface and HVAC (Heating, ventilation, and air condition) interface. Abdullah et al. [23] have used raptor codes in order to assess the relaying services along with using decode and forward scheme. A simulation-based scheme was adopted over conventional IEEE standard of VANET as well as design of MAC layer to find increase in its throughput. Another form of such non-conventional approach was carried out by Cheng and Trivedi [24] where gesture recognition is used for improving the driving experience over new form of hands-free infotainment system. The scheme make use of vision-based computational model where supervised learning scheme along with denoising scheme has been used for training the system to understand hand gestures. The work carried out by Galarza et al. [25] has presented an adaptive scheme to develop an interface for machine and human interaction in presence of dynamic scenarios of driving. A prototyping has been carried out for this purpose to check its statistical outcomes. However, direct applicability as well as work towards developing infotainment system is restricted only till this much of research papers and no further significant research work found till now.

\subsection{Integration cloud with vehicular network}

In order to support various services and application over the infotainment system over vehicular network, there is a potential need of a distributed and large network which is also ubiquitous in nature. Hence, cloud is one of the most preferred backbone or core network that can solve maximum problems associated with the accessing diversified services in vehicles [26]. As cloud offers extensive scheme for supporting various services, therefore studies towards combined usage of cloud and vehicular network is classified into three types on the basis of the existing research trends:

a. Schema-based Approaches: This kind of approaches makes use of unique schemes and planning in order to improve the service accessibility over vehicular network that can indirectly improve the performance of infotainment system. However, it should be known that such studies do not directly target towards infotainment system; however, their outcomes are claimed to offer better supportability towards challenging services accessed using infotainment system over vehicular network. Most recently, the work carried out by Shah et al. [27] discussed that transmit-power has prominent effect as well as it also has direct connectivity with the state of congestion in vehicular network. Hence, the solution offered by author was by using game theory for developing an adaptive scheme for congestion control considering transmit-power. It was also seen that usage of fog computing too offers advantage to vehicular cloud network. One such usage can be found in the work of Tang et al. [28] where fog computing is used for smart parking system using experimental approach over vehicular network. A typical algorithm is written for exploring the free parking slot using multiple optimization objects. The analysis of the study is carried out using cost over space of parking. However, the study doesn't 
consider many other essential elements e.g. information and domain related systems and is focused only on specific information. Emphasis on information as well as domain related schemes has been theoretically discussed by Grewe et al. [29] using different use cases. The study reveal that existing approaches using such schemes have diversified differences with respect to mechanism of data dissemination, naming, or security, etc; however, all the approaches offer equal emphasis to the mobility problems. Moreover, the impact of data offloading is not much found to be discussed in this work. It is believed that data offloading results in challenging situation of data explosion over wireless network and hence a control scheme is required. The study towards addressing this problem was carried out by Huang et al. [30] where an integration of mobile edge computing and software defined network has been utilized to develop a control scheme. This scheme allows a formulation of better route for vehicle-tovehicle communication scheme using contextual information. It should be noted that majority of such system is basically operational over IP network which doesn't offer suitability for any form of contentcentric network. Hussain et al. [31] claimed that if such IP network is connected with vehicular cloud network that such problems could be resisted. Existing system also offers a scheme for catering up demands of autonomous vehicles. The work carried out by Maalej et al. [32] uses computer vision in order to develop a multimodal scheme for identification of object by the vehicle followed by recognition of it. A hypothetical scheme towards improving vehicular cloud has been also emphasized by Gong et al. [33] and Huang et al. [34]. Study towards resource allocation for effective heterogeneous vehicle is carried out by Lin et al. [35] using Markov modeling. The work of Quan et al. [36] has emphasized on improving the delivery quality by packetizing the multimedia file content over vehicular cloud network. The authors have used Markov chain for system model. A storage scheme was presented by $\mathrm{Wu}$ et al. [37] where it targeted for transmitting the data to a different vehicular node prior to transmitting the data to particular zone. Fuzzy logic was utilized and cluster-based method is used for transmitting the data. The problem associated with the energy efficiency and the quality of experience is investigated by Xu et al. [38]. The work of Girau et al. [39] have presented a discussion of reusability of different layers considering IoT for developing a social platform of all the objects that are connected to each other. The work carried out by Kim et al. [40] has presented discussion on the solution towards the data forwarding scheme. The scheme uses greedy algorithm as well as learning scheme in order model data dissemination process. Li et al. [41] have presented a discussion of a scheme that uses smart phone for facilitating routing scheme on vehicular network. Adoption of game theory is also seen in the work of Zhang et al. [42] towards intelligent transportation system. The mechanism introduces a trajectory-based system to establish communication among the vehicles. A search-based approach is developed by Mershad and Artail [43] where an efficient cloud server is looked for.

b. Security-based Approaches: Owing to the utilization of the diversified adhoc network in highly distributed fashion, the communications within the vehicular nodes are highly vulnerable. Hence, security is of higher degree of concern when it comes to vehicular cloud network. The consequences of security breach can cost the life of a driver by remotely tampering the infotainment system. As infotainment system also retains various private information about the vehicle owner, hence privacy is always of higher concern. Most recently, the work carried out by Huang et al. [44] have addressed such security problems quite uniquely. A security schema is designed that is capable of resisting all sorts of negative messages or counterfeited messages. The work carried out by Yang et al. [45] have used game theory in order to compute the channel capacity associated with different vehicles for controlling the security breach and make the communication more efficient. Problems associated with the privacy problems are discussed by Xue et al. [46]. The authors have used encryption based mechanism using fog computing to perform prediction of mobility of vehicular nodes to fog server. Shao et al. [47] have presented a privacy protection scheme over vehicular cloud network by combining digital signature scheme and tree-based authentication mechanism. Another study over privacy problem is addressed by $\mathrm{Ni}$ et al. [48] where a navigation assistance system has been designed. The technique uses Bloom filter for retrieving the data for supporting querying system and a mathematical model is presented using digital signature which ensure validation and updating of all the vehicular nodes. Solution towards data stealing problems from infotainment system can be resisted of the vehicular communication is safeguarded with fail-proof mechanism. Study towards such direction was carried out by Luo and Ma [49] where multi-authority scheme of attribute based cryptography has been implemented for controlling access on the vehicles. Study towards emphasis on data security has been presented by Li et al. [50]. According to the study, over channel utilization, is one indicator of security breach and existing security approaches doesn't offer comprehensive security solution. The study carried out by Fan et al. [51] have discussed that mobility with high speed and distribution of node with high density can significantly affect data forwarding as well as may invite security problems too. Therefore, the solution presented by authors is based on retrieving the ciphertext with higher efficiency. 
The problem associated with the privacy problem is also addressed in the work of Hussain et al. [52]. An interesting solution has been presented by the author where images are captured from the site of interest by the image capturing devices of the vehicles and the information is forwarded to the cloud. The capturing of image is carried out only when the vehicular node encounters any event. An incentivebased mechanism is introduced in order to promote participation of many vehicles. The works carried out by Soleymani et al. [53] have presented a trust-based framework in order to maintain the reliability and integrity among the vehicular nodes. Harnessing fuzzy logic, the model is capable of checking the validity often messages exchanged by the vehicles. Usage of message authentication codes is also reported for ensuring better anonymity of the messages exchange in vehicular networks. The work of Jiang et al. [54] has used hash message authentication codes for performing authentication of list of revoked certificate. The implementation has used pseudonym for strengthening the privacy factor too. A unique study carried out by Zhou et al. [55] have used delay tolerant network in order to resist the node compromise attack. The study also shows that it can offer equal performance towards data delivery performance too apart from security. Another unique study was proposed by Liu et al. [56] where a downlink dissemination of the message is presented. According to the study, the delivery of the secured messages are offered with higher priority using specifically defined gateways followed by message dissemination among the vehicle.

c. Traffic-based Scheme: Traffic-based scheme is another approach which has been implemented by some of the researchers. The prime motive of this kind of approach is to basically to develop a unique topological policy which has a direct impact on the traffic system of vehicular network. Majority of such scheme make use of directionality factor and various conditional options for allowing the traffic. Most recently, there was no such approach and the only latest approach in this regard is found in the year 2016, where Azizian et al. [57] have presented a transmission scheduling. In such schemes sensor has been utilized for scheduling by using a cluster formation approach for newly forming vehicular cloud dynamically and not in predefined fashion. Calculation towards relative mobility is carried out for selecting the broker node. The approach uses medium access control using contention free approach. Usage of cooperative relaying also assists in formation of a new traffic system for an efficient service relaying over infotainment system in vehicular cloud networks. The work carried out by Feteiha and Hassanein [58] has discussed about the cooperative relaying mechanism over 4G networks. According to the authors, existing relaying scheme suffers from performance degradation that adversely affects the data arriving on the infotainment device while using existing vehicular cloud system. It also suffers from more degree of channel related errors. Therefore, the presented scheme uses cooperative technique with pre-coded scheme which is meant for extracting the spatial diversity information of $4 \mathrm{G}$ network. Adoption of this scheme is claimed to enhance the traffic system among the node communication. According to Salahuddin et al. [59], the contribution of software defined network is essential for framing up the traffic system of vehicular cloud. The authors have presented an architecture where a unique road side unit is developed for dynamic traffic management.

Therefore, it can be seen that there are various forms of algorithms as well as approaches which directly as well as indirectly assists in enriching the operation of infotainment system. The direct systems are developed towards infotainment system while the indirect systems are developed towards improving the vehicular cloud system which can directly contribute to support the better performance of infotainment system. Table 1 summarizes all the existing research contribution.

Table 1. Summary of existing research contribution

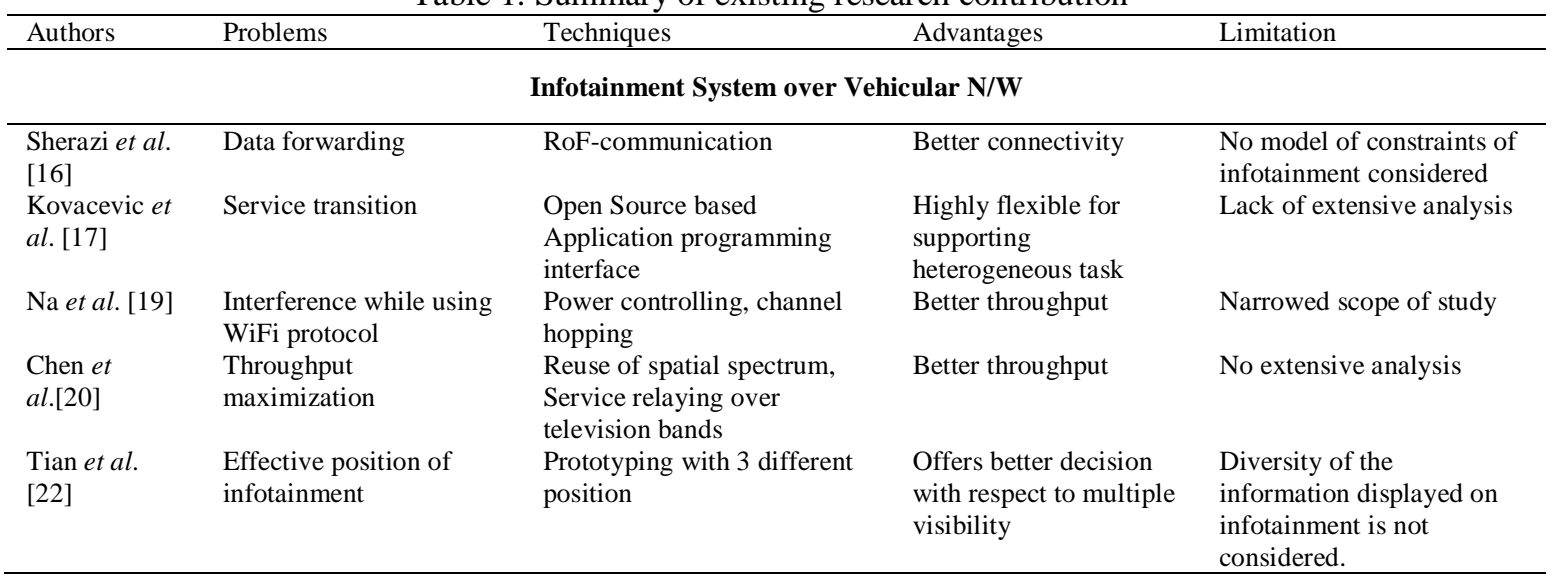


Table 1. Summary of existing research contribution (continue)

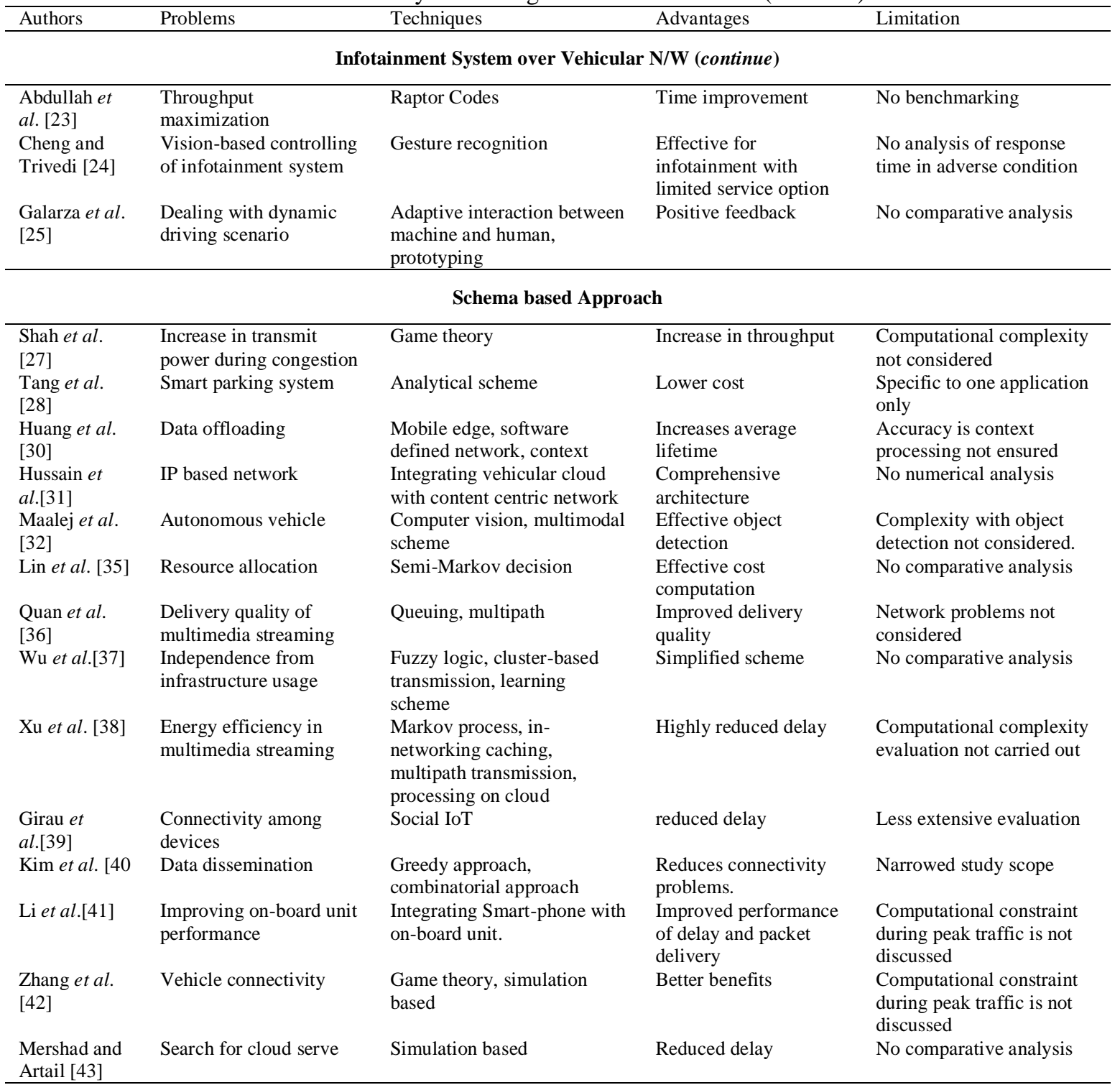

\section{Security-based Approach}

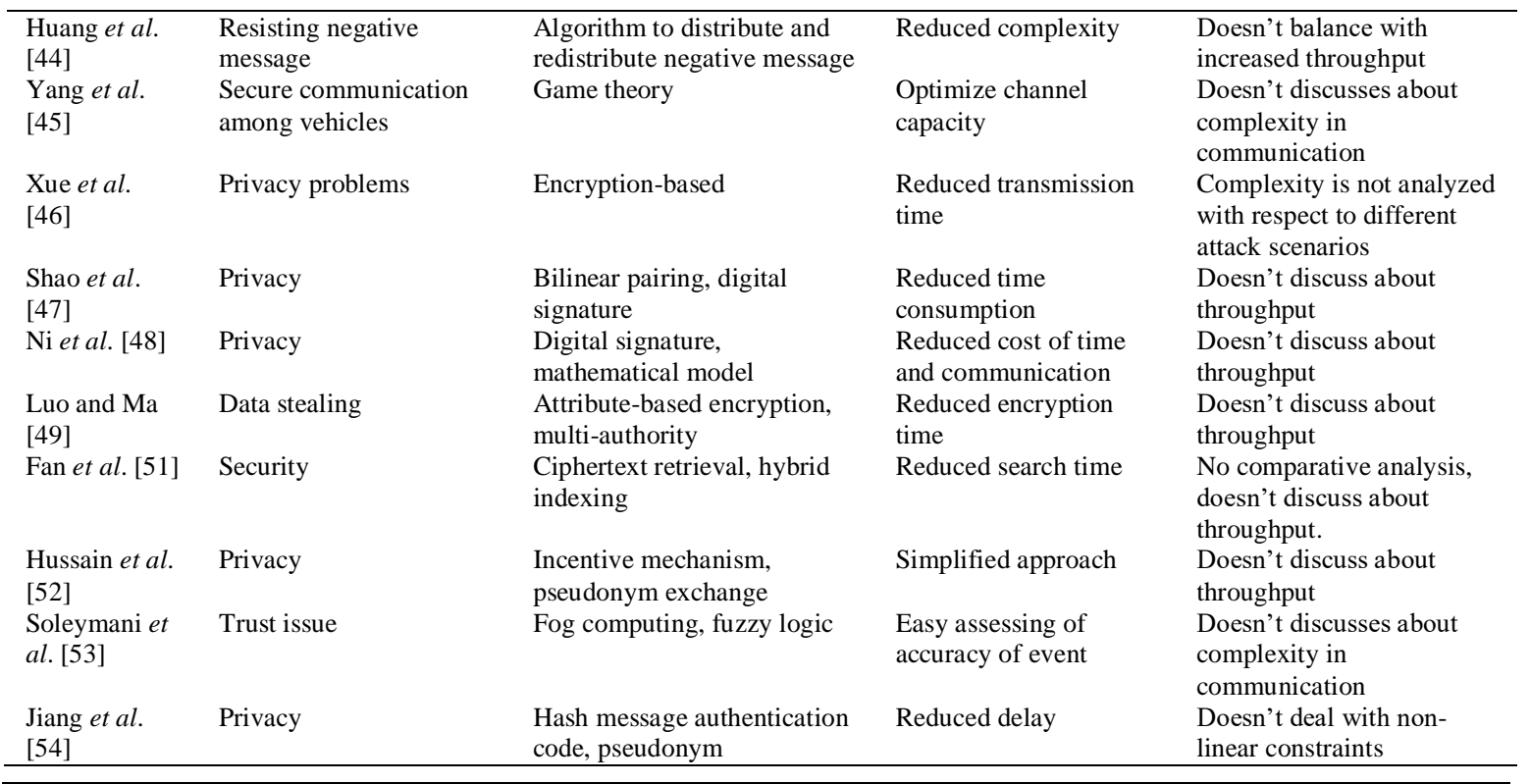




\begin{tabular}{|c|c|c|c|c|}
\hline Authors & Problems & Techniques & Advantages & Limitation \\
\hline \multicolumn{5}{|c|}{ Security-based Approach (continue) } \\
\hline $\begin{array}{l}\text { Zhou et al. } \\
\text { [55] }\end{array}$ & Privacy & $\begin{array}{l}\text { Delay tolerant network, } \\
\text { incentive scheme }\end{array}$ & Offers reliability & $\begin{array}{l}\text { Doesn't discusses about } \\
\text { complexity in } \\
\text { communication }\end{array}$ \\
\hline Liu et al. [56] & $\begin{array}{l}\text { Secure message } \\
\text { dissemination }\end{array}$ & $\begin{array}{l}\text { Gateway and target area, } \\
\text { selection of delegator node, } \\
\text { mathematical modeling }\end{array}$ & $\begin{array}{l}\text { Reduce delay, lower } \\
\text { overhead }\end{array}$ & $\begin{array}{l}\text { Doesn't discusses about } \\
\text { complexity in } \\
\text { communication }\end{array}$ \\
\hline \multicolumn{5}{|c|}{ Traffic-based Scheme } \\
\hline $\begin{array}{l}\text { Azizian et al. } \\
\text { [57] }\end{array}$ & Transmission scheduling & $\begin{array}{l}\text { Contention free medium } \\
\text { access control }\end{array}$ & $\begin{array}{l}\text { Stabilized performance } \\
\text { of newly formed } \\
\text { vehicular cloud }\end{array}$ & $\begin{array}{l}\text { Analysis doesn't consider } \\
\text { peak traffic condition }\end{array}$ \\
\hline $\begin{array}{l}\text { Feteiha and } \\
\text { Hassanein } \\
{[58]}\end{array}$ & Channel fading & $\begin{array}{l}\text { Cooperative transmission, } \\
\text { spatial diversity }\end{array}$ & $\begin{array}{l}\text { Lower outage } \\
\text { probability }\end{array}$ & Issues of LTE not modeled \\
\hline $\begin{array}{l}\text { Salahuddin et } \\
\text { al. [59] }\end{array}$ & $\begin{array}{l}\text { Cloud resource } \\
\text { management }\end{array}$ & $\begin{array}{l}\text { A new roadside unit, } \\
\text { reinforcement learning }\end{array}$ & $\begin{array}{l}\text { Minimized cost of } \\
\text { configuration, reduced } \\
\text { delay }\end{array}$ & $\begin{array}{l}\text { Complexity associated in } \\
\text { peak traffic not analyzed. }\end{array}$ \\
\hline
\end{tabular}

\section{OPEN END PROBLEMS OR RESEARCH GAPS}

There is no denying the fact that existing research contribution discussed in prior section has given a considerable edge of features towards infotainment system. However, majority of the problems are associated with the loopholes which are yet to be investigated. Following are some briefing of the open end problems extracted after reviewing the existing system:

a. Bias focus on Problems: In order to offer a superior form of service relaying on infotainment system, it is required that existing research contribution should offer better quality of service, flexible form of modeling, resistivity capability against all form of potential threats, and capability to deal with all sorts of traffic / network related issues. Unfortunately, majority of the research work are focused on security problems and least on other quality of service problems. The operation carried out by an infotainment system demands a complete supportability of vehicular cloud network which has many other problems critical than security problems. Even from security solution discussed, they are not meant for dynamic adversarial attack but are quite specific to one form of attacks. Moreover, maximum approaches have no evidence that they are also effective towards data delivery performance along with security. Hence, there is a long way to go ahead even to obtain a better form of security solution.

b. Doesn't Cater up Actual Demand of Infotainment System: A robust and better form of infotainment system can be only said if they have complete support of minimal latency, offers optimal bandwidth, and maximum retention of connectivity among the vehicles. Although, there are many literatures that claims of lower delay, but they are not found to claim for other performance factors which are equally essential to prove the supportability of futuristic infotainment system. Without lower latency, it is not feasible to support delivery of massive streams of contents while maximum channel capacity is required for seamless uploading of necessary back end services and data to offer online loading of dynamic services. Similarly, seamless connectivity is highly essential as infotainment system for upcoming driverless vehicle requires non-intermittent and fail-proof connectivity. There is no such literature where all these problems are sorted out in reality.

c. Lack of consideration of Infotainment Device: Basically, an infotainment device is a part of the on-board unit in intelligent transportation system. In order to offer better service supportability with better quality of experience, it is required that all the capability factor associated with radio communication of the vehicle should be considered e.g. adopted channels for radio frequency, radio communication using single/multiple hops, bit rate, available channel capacity, degree of compensation towards propagation issues for radio signals, etc. In existing system, all these factors are considered only for road side units and never for on-board units.

d. Non-Applicability to Road Transport System: A closer look into various scheme-based approach as well as traffic-based approach shows good conceptual model but without any evidence of their applicability to road transport system. It was seen that majority of the research contribution under this uses data dissemination process using unicast and broadcasting scheme. The unicast mechanism uses trajectorybased, stability factor of link, and geographic-based. Such approaches results in vehicle to fail in exploring the next vehicular node to perform forwarding because network related global knowledge is not provided to the vehicles. For this, the data dissemination schemes are not equipped with global data. 
Apart from this, existing approaches of data dissemination on vehicular cloud are carried out on specific network model which is pre-configured.

e. Unrealistic Test Environment \& Modeling: Basically, the service to support infotainment requires massive flow of information. Therefore, real-time usage of infotainment system is shrouded with different challenges due to maximum transmission rate of data along with adaptability of dynamic topology. This requires a joint accomplishment of interference-free network, higher bandwidth, lower resource consumption, etc. Unfortunately, all these are hard to achieve at same time as per existing approaches without which it is not feasible for achieve superior quality of streamed data. Existing studies towards multimedia streaming doesn't deal with contention problem as well as they also lack resisting problems like congestion and collision.

f. Lack of Optimization Approach: There are no research works carried out towards optimization. At present, there are various optimization algorithms dealing with wide range of scientific problems. Even in cloud computing, machine learning is slowly increasing in almost all the application to offer better optimized outcomes in order to meet user demands. Unfortunately, the research work using optimization based approach is significantly missing which has capability to solve maximum problems. There is an immense need of novel optimization technique to find best solution for supportability of seamless services on infotainment devices using vehicular cloud network.

\section{CONCLUSION}

This manuscript discusses about the actual scenario of infotainment system in vehicular network. After reviewing the existing studies it is just concluded that there are lot of scope of work to be carried out over vehicular cloud system with more emphasis on comprehensive computational model. Existing work can be just treated as a better form of guidelines to start a new work with more exposure towards achievements as well as limitation. The contribution of the this manuscript is that it offers better idea of Advancement in Infotainment System in Automotive Sector with Vehicular Cloud Network by introdcing the basic fundamentals of the infotainment system along with gaps in the research area of Automotive Sector with Vehicular Cloud Network. The future work could be on the direction of the addressing the open end issues discussed in prior section. A novel computational model can be constructed with inclusion of real-time non-linear constraints so that maximum problems associated with robust and efficient service supportability towards infotainment system can be developed.

\section{REFERENCES}

[1] M. Traub, A. Maier and K. L. Barbehön, "Future Automotive Architecture and the Impact of IT Trends," in IEEE Software, vol. 34, no. 3, pp. 27-32, May-Jun. 2017.

[2] Felipe Jimenez, "Intelligent Vehicles: Enabling Technologies and Future Developments," Butterworth-Heinemann, 2017.

[3] Ammar Rayes, Samer Salam, "Internet of Things From Hype to Reality: The Road to Digitization," Springer, 2018.

[4] Markus Mueck, Ingolf Karls, "Networking Vehicles to Everything: Evolving Automotive Solutions," Walter de Gruyter GmbH \& Co KG, 2018.

[5] Anna Maria Vegni, Valeria Loscrì, Athanasios V. Vasilakos, "Vehicular Social Networks," CRC Press, 2017.

[6] Chimata, Raghuveer, Singh, Rajesh, Singh, Bhupendra, "Internet of Things in Automotive Industries and Road Safety," River Publishers, 2018.

[7] K. M. Alam, M. Saini and A. E. Saddik, "Toward Social Internet of Vehicles: Concept, Architecture, and Applications," in IEEE Access, vol. 3, pp. 343-357, 2015.

[8] S. Céspedes and X. Shen, "A framework for ubiquitous IP communications in vehicle to grid networks," 2011 IEEE GLOBECOM Workshops (GC Wkshps), Houston, TX, pp. 1231-1235, 2011.

[9] C. Jeremiah and A. J. Nneka, "Issues and possibilities in Vehicular Ad-hoc Networks (VANETs)," 2015 International Conference on Computing, Control, Networking, Electronics and Embedded Systems Engineering (ICCNEEE), Khartoum, pp. 254-259, 2015.

[10] L. Aparecido, "Data dissemination in vehicular networks: Challenges, solutions, and future perspectives," 2015 7th International Conference on New Technologies, Mobility and Security (NTMS), Paris, pp. 1-5, 2015.

[11] G. Samara, W. A. H. Al-Salihy and R. Sures, "Security issues and challenges of Vehicular Ad Hoc Networks (VANET)," 4th International Conference on New Trends in Information Science and Service Science, Gyeongju, pp. 393-398, 2010.

[12] S. M. Hanshi and M. M. Kadhum, "Geographic routing protocol issues in vehicular ad hoc networks," 2013 IEEE International Conference on RFID-Technologies and Applications (RFID-TA), Johor Bahru, pp. 1-7, 2013.

[13] Rakesh Shrestha, Rojeena Bajracharya, and Seung Yeob Nam, "Challenges of Future VANET and Cloud-Based Approaches," Hindawi, Wireless Communications and Mobile Computing, vol. 2018, 2018

[14] https://www.marketsandmarkets.com/Market-Reports/in-car-vehicle-infotainment-ici-systems-market-538.html

\footnotetext{
Advancement in infotainment system in automotive sector with vehicular cloud network and ... (Reshma S.)
} 
[15] Rasheed Hussain, Zeinab Rezaeifar, Heekuck Oh, "A Paradigm Shift from Vehicular Ad Hoc Networks to VANET-Based Clouds," Springer Journal, wireless Personal COmmunication, 2015

[16] Sherazi, Hafiz Husnain Raza, Zuhaib Ashfaq Khan, Razi Iqbal, Shahzad Rizwan, Muhammad Ali Imran, and Khalid Awan. "A Heterogeneous IoV Architecture for Data Forwarding in Vehicle to Infrastructure Communication," Mobile Information Systems, vol. 2019, 2019.

[17] Kovacevic, Branimir, Marko Kovacevic, Tomislav Maruna, and Istvan Papp. "A java application programming interface for in-vehicle infotainment devices," IEEE Transactions on Consumer Electronics, vol. 63, no. 1, pp: 68-76, 2017.

[18] Guo, Jie, Bin Song, Ying He, Fei Richard Yu, and Mehdi Sookhak. "A survey on compressed sensing in vehicular infotainment systems," IEEE Communications Surveys \& Tutorials, vol. 19, no. 4, pp: 2662-2680, 2017.

[19] Na, Woongsoo, Nhu-Ngoc Dao, and Sungrae Cho, "Mitigating WiFi interference to improve throughput for in-vehicle infotainment networks." IEEE Wireless Communications, vol. 23, no. 1, pp: 22-28, 2016.

[20] Chen, Jiacheng, Bo Liu, Haibo Zhou, Lin Gui, Ning Liu, and Yiyan Wu, "Providing vehicular infotainment service using vhf/uhf tv bands via spatial spectrum reuse," IEEE transactions on broadcasting, vol. 61, no. 2, pp: 279-289, 2015.

[21] Rene, Sergi, Juanjo Alins, Jorge Mata-Díaz, Carlos Ganan, Jose L. Muñoz, and Oscar Esparza. "Vespa: Emulating infotainment applications in vehicular networks," IEEE pervasive computing, vol. 13, no. 3, pp: 58-66, 2014.

[22] Tian, Renran, Lingxi Li, Vikram S. Rajput, Gerald J. Witt, Vincent G. Duffy, and Yaobin Chen. "Study on the display positions for the haptic rotary device-based integrated in-vehicle infotainment interface," IEEE Transactions on Intelligent Transportation Systems, vol. 15, no. 3, pp: 1234-1245, 2014.

[23] Abdullah, Nor Fadzilah, Angela Doufexi, and Robert J. Piechocki. "Raptor codes-aided relaying for vehicular infotainment applications," IET Communications, vol. 7, no. 18, pp: 2064-2073, 2013.

[24] Cheng, Shinko Y., and Mohan M. Trivedi. "Vision-based infotainment user determination by hand recognition for driver assistance," IEEE transactions on intelligent transportation systems, vol. 11, no. 3, pp: 759-764, 2010.

[25] Galarza, Miguel Angel, Teresa Bayona, and Josep Paradells. "Integration of an adaptive infotainment system in a vehicle and validation in real driving scenarios," International journal of vehicular technology, vol. 2017, 2017.

[26] E. Lee, E. Lee, M. Gerla and S. Y. Oh, "Vehicular cloud networking: architecture and design principles," in IEEE Communications Magazine, vol. 52, no. 2, pp. 148-155, February 2014

[27] S. A. A. Shah, E. Ahmed, J. J. P. C. Rodrigues, I. Ali and R. Md Noor, "Shapely Value Perspective on Adapting Transmit Power for Periodic Vehicular Communications," in IEEE Transactions on Intelligent Transportation Systems, vol. 19, no. 3, pp. 977-986, March 2018.

[28] C. Tang, X. Wei, C. Zhu, W. Chen and J. J. P. C. Rodrigues, "Towards Smart Parking Based on Fog Computing," in IEEE Access, vol. 6, pp. 70172-70185, 2018.

[29] D. Grewe, M. Wagner and H. Frey, "A Domain-Specific Comparison of Information-Centric Networking Architectures for Connected Vehicles," in IEEE Communications Surveys \& Tutorials, vol. 20, no. 3, pp. 2372-2388, thirdquarter 2018.

[30] C. Huang, M. Chiang, D. Dao, W. Su, S. Xu and H. Zhou, "V2V Data Offloading for Cellular Network Based on the Software Defined Network (SDN) Inside Mobile Edge Computing (MEC) Architecture," in IEEE Access, vol. 6, pp. 17741-17755, 2018.

[31] R. Hussain, S. H. Bouk, N. Javaid, A. M. Khan and J. Lee, "Realization of VANET-Based Cloud Services through Named Data Networking," in IEEE Communications Magazine, vol. 56, no. 8, pp. 168-175, August 2018.

[32] Maalej, Yassine, Sameh Sorour, Ahmed Abdel-Rahim, and Mohsen Guizani. "Vanets meet autonomous vehicles: Multimodal surrounding recognition using manifold alignment," IEEE Access, vol. 6, pp: 29026-29040, 2018.

[33] Gong, Haigang, Lingfei Yu, Nianbo Liu, and Xue Zhang. "Mobile content distribution with vehicular cloud in urban VANETs," China Communications, vol. 13, no. 8, pp: 84-96, 2016.

[34] Huang, Xianan, Ding Zhao, and Huei Peng. "Empirical study of dsrc performance based on safety pilot model deployment data," IEEE Transactions on Intelligent Transportation Systems, vol. 18, no. 10, pp: 2619-2628, 2017.

[35] Lin, Chun-Cheng, Der-Jiunn Deng, and Chia-Chi Yao. "Resource allocation in vehicular cloud computing systems with heterogeneous vehicles and roadside units," IEEE Internet of Things Journal, vol. 5, no. 5, pp: 3692-3700, 2018.

[36] Quan, Wei, Fei Song, Chengxiao Yu, and Mingchuan Zhang. "ICN based vehicle-to-cloud delivery for multimedia streaming in urban vehicular networks," China Communications, vol. 13, no. 9, pp: 103-112, 2016.

[37] Wu, Celimuge, Tsutomu Yoshinaga, Yusheng Ji, Tutomu Murase, and Yan Zhang. "A reinforcement learningbased data storage scheme for vehicular ad hoc networks," IEEE Transactions on Vehicular Technology, vol. 66, no. 7, pp: 6336-6348, 2017.

[38] Xu, Changqiao, Wei Quan, Hongke Zhang, and Luigi Alfredo Grieco. "GrIMS: Green information-centric multimedia streaming framework in vehicular ad hoc networks," IEEE Transactions on Circuits and Systems for Video Technology, vol. 28, no. 2, pp: 483-498, 2018.

[39] Girau, Roberto, Salvatore Martis, and Luigi Atzori. "Lysis: A platform for IoT distributed applications over socially connected objects," IEEE Internet of Things Journal, vol. 4, no. 1, pp: 40-51, 2017.

[40] Kim, Ryangsoo, Hyuk Lim, and Bhaskar Krishnamachari. "Prefetching-based data dissemination in vehicular cloud systems," IEEE Transactions on Vehicular Technology, vol. 65, no. 1, pp: 292-306, 2016.

[41] Li, Xiuqi, Yong Feng, Feng Wang, and Qian Qian. "When smart phone meets vehicle: A new on-board unit scheme for VANETs," In 2015 IEEE International Conference on Computer and Information Technology; Ubiquitous 
Computing and Communications; Dependable, Autonomic and Secure Computing; Pervasive Intelligence and Computing, pp. 1095-1100. IEEE, 2015.

[42] Zhang, Lin, Shucong Jia, Zishan Liu, Yumei Wang, and Yu Liu, "Bus-Ads: Bus trajectory-based advertisement distribution in VANETs using coalition formation games," IEEE Systems Journal, vol. 11, no. 3, pp: 1259-1268, 2017.

[43] Mershad, Khaleel, and Hassan Artail, "Finding a STAR in a Vehicular Cloud," IEEE Intelligent transportation systems magazine, vol. 5, no. 2, pp: 55-68, 2013.

[44] Huang, Baohua, Xiaolu Cheng, Caixia Huang, and Wei Cheng. "Meet-cloud for secure and accurate distribution of negative messages in vehicular Ad hoc network," Tsinghua Science and Technology, vol. 23, no. 4, pp: 377-388, 2018.

[45] Yang, Yixian, Xinxin Niu, Lixiang Li, and Haipeng Peng. "A Secure and Efficient Transmission Method in Connected Vehicular Cloud Computing," IEEE Network, vol. 32, no. 3, pp: 14-19, 2018.

[46] Xue, Kaiping, Jianan Hong, Yongjin Ma, David SL Wei, Peilin Hong, and Nenghai Yu. "Fog-aided verifiable privacy preserving access control for latency-sensitive data sharing in vehicular cloud computing," IEEE Network, vol. 32, no. 3, pp: 7-13, 2018.

[47] Shao, Bilin, Genqing Bian, Yue Wang, Shenghao Su, and Cheng Guo. "Dynamic Data Integrity Auditing Method Supporting Privacy Protection in Vehicular Cloud Environment," IEEE Access, vol. 6, pp: 43785-43797, 2018.

[48] Ni, Jianbing, Kuan Zhang, Yong Yu, Xiaodong Lin, and Xuemin Shen. "Privacy-preserving smart parking navigation supporting efficient driving guidance retrieval," IEEE Transactions on Vehicular Technology, vol. 67, no. 7, pp: $6504-6517,2018$

[49] Luo, Wei, and Wenping Ma. "Efficient and Secure Access Control Scheme in the Standard Model for Vehicular Cloud Computing," IEEE Access, vol. 6, pp: 40420-40428, 2018.

[50] Li, Hongwei, Rongxing Lu, Jelena Misic, and Mohamed Mahmoud. "Security and privacy of connected vehicular cloud computing," IEEE Network, vol. 32, no. 3, pp: 4-6, 2018.

[51] Fan, Kai, Xin Wang, Katsuya Suto, Hui Li, and Yintang Yang. "Secure and efficient privacy-preserving ciphertext retrieval in connected vehicular cloud computing," IEEE Network, vol. 32, no. 3, pp: 52-57, 2018.

[52] Hussain, Rasheed, Donghyun Kim, Junggab Son, Jooyoung Lee, Chaker Abdelaziz Kerrache, Abderrahim Benslimane, and Heekuck Oh. "Secure and Privacy-Aware Incentives-Based Witness Service in Social Internet of Vehicles Clouds," IEEE Internet of Things Journal, vol. 5, no. 4, pp: 2441-2448, 2018.

[53] Soleymani, Seyed Ahmad, Abdul Hanan Abdullah, Mahdi Zareei, Mohammad Hossein Anisi, Cesar VargasRosales, Muhammad Khurram Khan, and Shidrokh Goudarzi. "A secure trust model based on fuzzy logic in vehicular ad hoc networks with fog computing," IEEE Access, vol. 5, 5619-15629, 2017.

[54] Jiang, Shunrong, Xiaoyan Zhu, and Liangmin Wang. "An efficient anonymous batch authentication scheme based on HMAC for VANETs," IEEE Transactions on Intelligent Transportation Systems, vol. 17, no. 8, pp: 2193-2204, 2016.

[55] Zhou, Jun, Xiaolei Dong, Zhenfu Cao, and Athanasios V. Vasilakos. "Secure and privacy preserving protocol for cloud-based vehicular DTNs," IEEE Transactions on Information Forensics and Security, vol. 10, no. 6, pp: 1299-1314, 2015.

[56] Liu, Bingyi, Dongyao Jia, Jianping Wang, Kejie Lu, and Libing Wu. "Cloud-assisted safety message dissemination in VANET-cellular heterogeneous wireless network," IEEE systems journal, vol. 11, no. 1, pp: 128-139, 2017.

[57] Azizian, Meysam, Soumaya Cherkaoui, and Abdelhakim Hafid. "An optimized flow allocation in vehicular cloud," IEEE Access, vol. 4, pp: 6766-6779, 2016.

[58] Feteiha, Mohamed F., and Hossam S. Hassanein. "Enabling cooperative relaying VANET clouds over LTE-A networks," IEEE transactions on vehicular technology, vol. 64, no. 4, pp: 1468-1479, 2015.

[59] Salahuddin, Mohammad Ali, Ala Al-Fuqaha, and Mohsen Guizani. "Software-defined networking for rsu clouds in support of the internet of vehicles," IEEE Internet of Things journal, vol. 2, no. 2, pp: 133-144, 2015.

\section{BIOGRAPHIES OF AUTHORS}

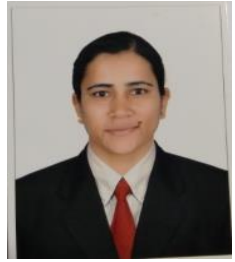

Reshma S. received her degree in Computer Science and Engineering from UBDT college of Engineering (2001)-Davanagere. She received her masters (M.Tech) degree from BIET (2009)Davanagere. She worked as Lecturer in Govt. Polytechnic college (2002), BIET (2002-2005) and STJIT (2005-2009). Currently, she is working as Assistant professor in Global Academy of Technology (from 2011), Bangalore. She had been counselor in IGNOU for two years. She has authored or published 2 publications. Her areas of interest are Networking and cloud computing.

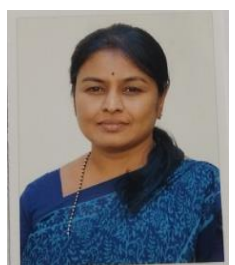

Dr. Chetanaprakash had been Board of Studies member in VTU (2016-2019), BOE member in VTU and Kuvempu University, working as NODAL officer for AISHE institute and Dept. Web-coordinator. She has been session chair for IWSSIP, South-East Europe (2011), session chair and reviewer in iCATccT-2015. She has authored or published ...publications. Her area of interest are analysis \& design of algorithms, Discrete mathematical structures, Speech Signal Processing, Image processing, Time frequency analysis, ANN techniques etc. 